\title{
Polariton-polariton interactions and stimulated scattering in semiconductor microcavities
}

\author{
M.S. Skolnick ${ }^{\text {a, }}$, R.M. Stevenson ${ }^{\text {a }}$, A.I. Tartakovskii ${ }^{\text {a }}$, R. Butté ${ }^{\text {a }}$, M. Emam-Ismail ${ }^{\text {a }}$, \\ D.M. Whittaker ${ }^{\text {b }}$, P.G. Savvidis ${ }^{\text {c }}$, J.J. Baumberg ${ }^{c}$, A. Lemaître ${ }^{a}$, V.N. Astratov ${ }^{a}$, \\ J.S. Roberts ${ }^{\mathrm{d}}$ \\ ${ }^{a}$ Department of Physics and Astronomy, University of Sheffield, Sheffield S3 7RH, UK \\ ${ }^{\mathrm{b}}$ Toshiba Research Europe Ltd., Cambridge CB4 4WE, UK \\ ${ }^{\mathrm{c}}$ Department of Physics and Astronomy, University of Southampton, Southampton SO17 1BJ, UK \\ d Department of Electronic and Electrical Engineering, University of Sheffield, Sheffield S1 3JD, UK
}

\begin{abstract}
Recent work on polariton-polariton scattering in semiconductor microcavities under continuous wave excitation conditions is reviewed. For weak non-resonant laser excitation, a marked bottleneck in the polariton distribution is observed, but which is suppressed by polariton-polariton scattering as the laser intensity is increased. However, the high excitation conditions necessary to observe stimulated emission lead to loss of strong coupling and conventional lasing in the weak coupling regime. By contrast for resonant excitation, polaritons are created directly in the polariton trap formed by the microcavity dispersion curve. Stimulated scattering of the bosonic quasi-particles occurs to the emitting state at the centre of the Brillouin zone, and to a companion state at high wavevector. The stimulation phenomena lead to condensation of the bosonic quasi-particles to two specific regions of k-space, and to the formation of a new state with macroscopic coherence. The prospects to achieve a polariton laser under conditions of non-resonant excitation are discussed. (C) 2002 Elsevier Science B.V. All rights reserved.
\end{abstract}

Keywords: Microcavities; Exciton-polaritons; Bosonic stimulation; Final state stimulation

\section{Introduction}

Semiconductor microcavities are Fabry-Pérot cavities containing quantum wells embedded within the cavity region at antinodes of the confined optical field [1]. Such structures enable control of the properties of both excitons and photons in the same structure, and to a considerable extent also of the interaction between excitons and photons. Most importantly for the new physics described here, the dispersion relations of the resulting coupled excitonphoton modes, exciton-polaritons (termed cavity polaritons in the present case), can be engineered to produce a variety of new phenomena, including final state stimulation, a new condensed phase with macroscopic coherence, and very low threshold optical parametric oscillators.

\footnotetext{
* Corresponding author. Tel.: +44-1142-224277; fax: +44-1142728079.

E-mail address: m.skolnick@sheffield.ac.uk (M.S. Skolnick).
}

A schematic diagram of a typical structure employed in the present work is shown in Fig. 1. The structure consists of a $3 \lambda / 2 \mathrm{GaAs}$ cavity ( $\lambda$ is the wavelength of light in the medium) surrounded by 20 (below) and 17 (above) layers of $\mathrm{Al}_{0.13} \mathrm{Ga}_{0.87} \mathrm{As}-\mathrm{AlAs}$ high reflectivity Bragg mirrors. The cavity leads to quantisation of the optical field in the vertical direction, with free propagation within the plane, leading to the approximately quadratic photon dispersion shown in Fig. 2. Two sets of three $\operatorname{In}_{0.06} \mathrm{Ga}_{0.94}$ As quantum wells within the cavity lead to quantised excitonic states in the vertical direction with free exciton motion within the plane. The exciton and photon states are coupled together (the strong coupling limit) [2] leading to the formation of new quasi-particles, termed cavity (exciton)-polaritons, with dispersion relations possessed by neither photons nor excitons alone. Most notably the lower polariton branch exhibits a dispersion which is photon-like at small wavevector and exciton-like at large wavevector, with a point of inflection in the dispersion between these two extremes, as shown in Fig. 2. It is this dispersion, which is furthermore controllable by design, which leads to much of 


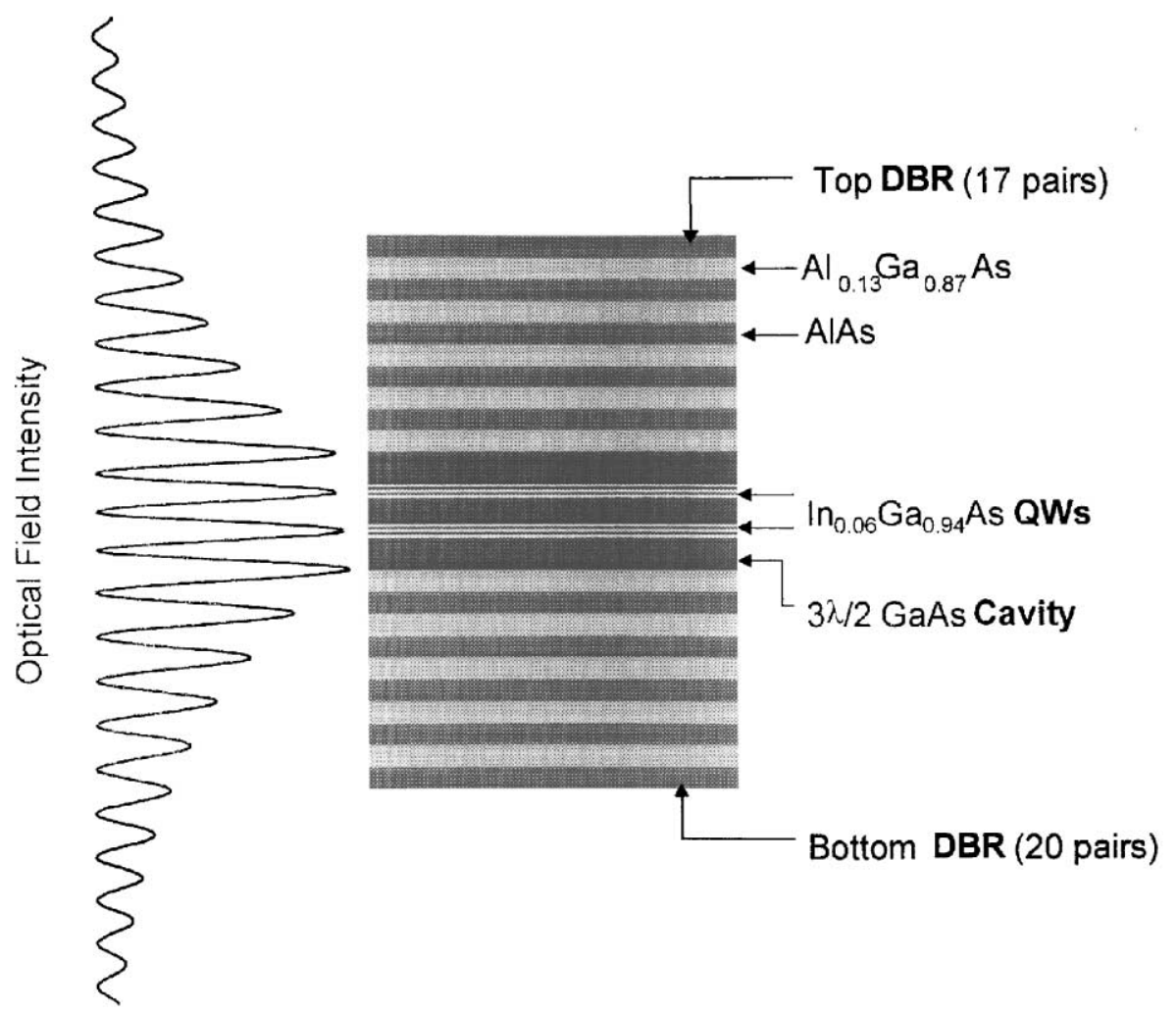

Fig. 1. Schematic diagram of microcavity structure containing two pairs of three quantum wells at the antinode of the optical field.

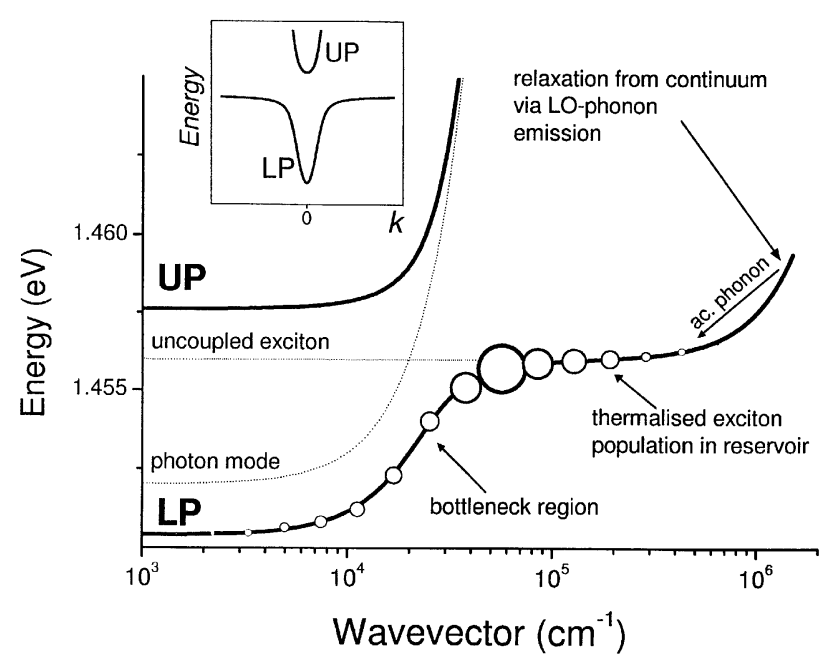

Fig. 2. Schematic diagram of lower and upper polariton branches in the strong coupling regime for $k$ values $<10^{7} \mathrm{~m}^{-1}$ and uncoupled exciton states for higher $k$ values (the exciton reservoir). For conditions of non-resonant excitation, the photocreated excitons populate the exciton reservoir, before scattering down into the polariton region. At low densities, a polariton relaxation bottleneck occurs close to the resonance point of the dispersion curves, as shown schematically on the figure, where the size of the symbols are proportional to occupancy. The dashed lines indicate the uncoupled exciton and photon modes. The inset shows the polariton region of the main part of the figure, but now plotted on a linear $k$ scale. The trap for polaritons formed by the microcavity dispersion is now easily visualised. the new physics reported in the present paper. It gives rise to a trap for polaritons (a trap in $k$-space as opposed to the real-space traps employed in atomic physics to study condensation phenomena) as shown in the inset to Fig. 2, whose depth can be varied by changing the detuning $\Delta$ (the energy separation) between the uncoupled exciton and photon modes. It also permits new energy and momentum conserving polariton-polariton scattering processes, not possible for either excitons or photons alone. Resonant excitation of the polariton states enables high state occupancies to be achieved, the observation of bosonic stimulation effects and the creation of polariton condensates [3-7].

The results are contrasted with those obtained by high energy non-resonant excitation, where for similar detunings a relaxation bottleneck is observed at low density, rapidly suppressed by exciton-exciton scattering. However, in this case, strong exciton broadening is observed, with the loss of the strong coupling limit before gain occurs. In this case stimulated emission is again observed, but now from a photon laser, as opposed to emission from the polariton states in the case of resonant excitation. The prospects for creating a 'polariton laser' under conditions of non-resonant excitation are discussed.

The paper is organised in the following way. The general type of experimental setup employed is first described, followed by the results for non-resonant excita- 


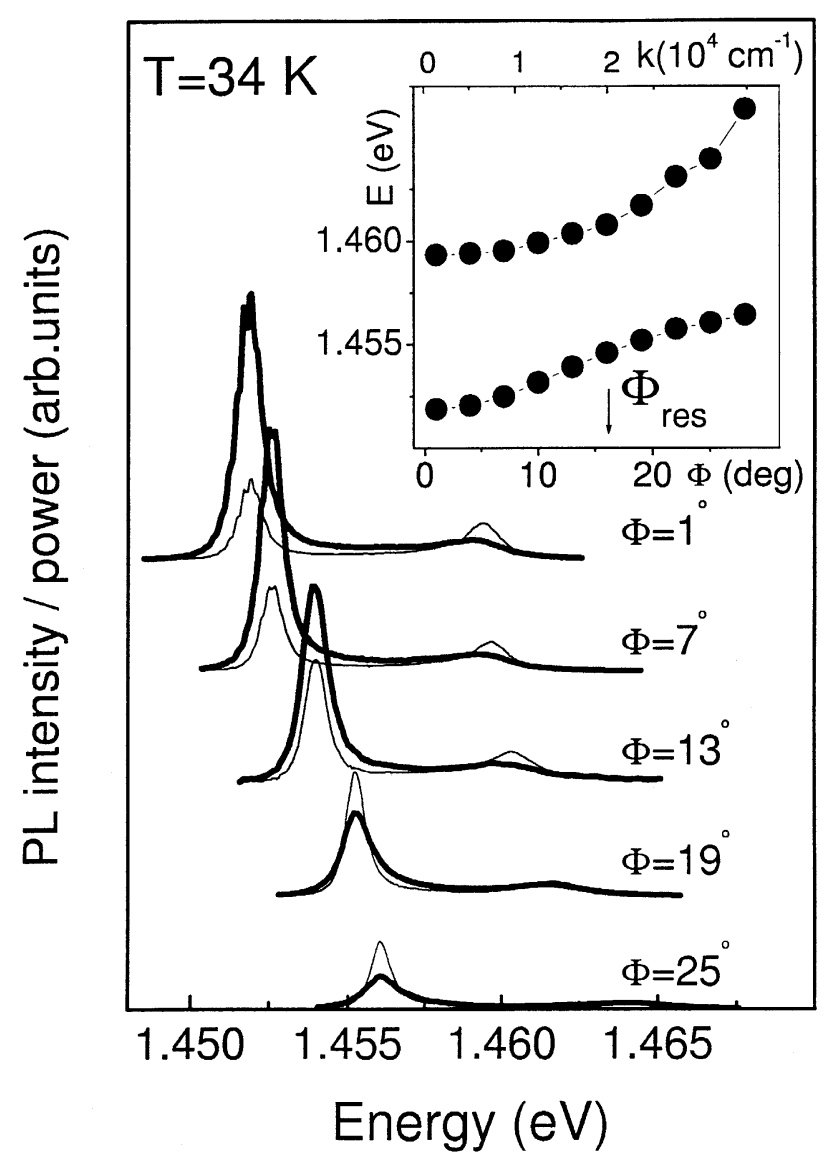

Fig. 3. Angle-resolved PL spectra at $34 \mathrm{~K}$ for non-resonant excitation at 5 $\mathrm{W} / \mathrm{cm}^{2}$ (thin lines) and $80 \mathrm{~W} / \mathrm{cm}^{2}$ (thick lines). The inset shows the measured polariton dispersion for the detuning of $-4.3 \mathrm{meV}$.

tion. The observation of stimulated scattering for resonant excitation is then presented and finally the non-resonant and resonant excitation results are compared and the main points of the paper are summarised.

\section{Experimental arrangement}

The experiments were carried out in cryostats with wide angular access in both excitation and detection channels, with angular resolutions in both cases of $\sim 1^{\circ}$. The ability to vary independently the angles of excitation and detection is a key point of this work. Since in-plane wavevector $k$ is related to $\Phi$ by $k=(w / c) \sin \Phi$, polaritons can be injected at specific points of the polariton dispersion by varying the angle of incidence of the laser, and the polariton occupation can be probed directly by varying the angle of detection. The one-to-one correspondence between $k$ and $\Phi$ for the polariton dispersion is a key point in microcavity physics as opposed to that of polaritons in bulk semiconductors [1]. Essentially, since the vertical wavevector is quantised in microcavities for both excitons and photons, and as the photons and hence the polaritons have a finite lifetime in the cavity, polaritons can be excited and probed directly in well-controlled experiments.

\section{Non-resonant excitation}

Photoluminescence spectra excited using non-resonant excitation at an energy $(1.56 \mathrm{eV})$ above the stop-band of the Bragg mirrors, are shown in Fig. 3, for angles of detection from $0^{\circ}$ to $25^{\circ}$ for two excitation densities of 5 and $80 \mathrm{~W} / \mathrm{cm}^{2}$ (thin and thick lines, respectively). The detuning employed is $-4 \mathrm{meV}$. PL signals from both lower and upper polariton branches are observed. Most notably the signal from the lower polariton branch at 5 $\mathrm{W} / \mathrm{cm}^{2}$ is observed to peak at an angle of $\sim 20^{\circ}$ before decreasing to higher angle. This is a signature of the relaxation bottleneck for polaritons, first discussed for bulk materials (see, e.g. Ref. [8]), and then for microcavity polaritons by Tassone and co-workers $[9,10]$, before its definitive observation for III-V [11] and II-VI [12] microcavities, respectively. Under the non-resonant excitation conditions employed, the photocreated excitons first relax rapidly by LO phonon relaxation $(<1 \mathrm{ps})$, followed by slower acoustic phonon emission to populate the high density of high $k$ exciton states (the exciton reservoir), as indicated schematically in Fig. 2. The excitons then relax from the reservoir into the region of strongly coupled polariton states, as also indicated schematically on Fig. 2 . The bottleneck arises as a result of the competition be-

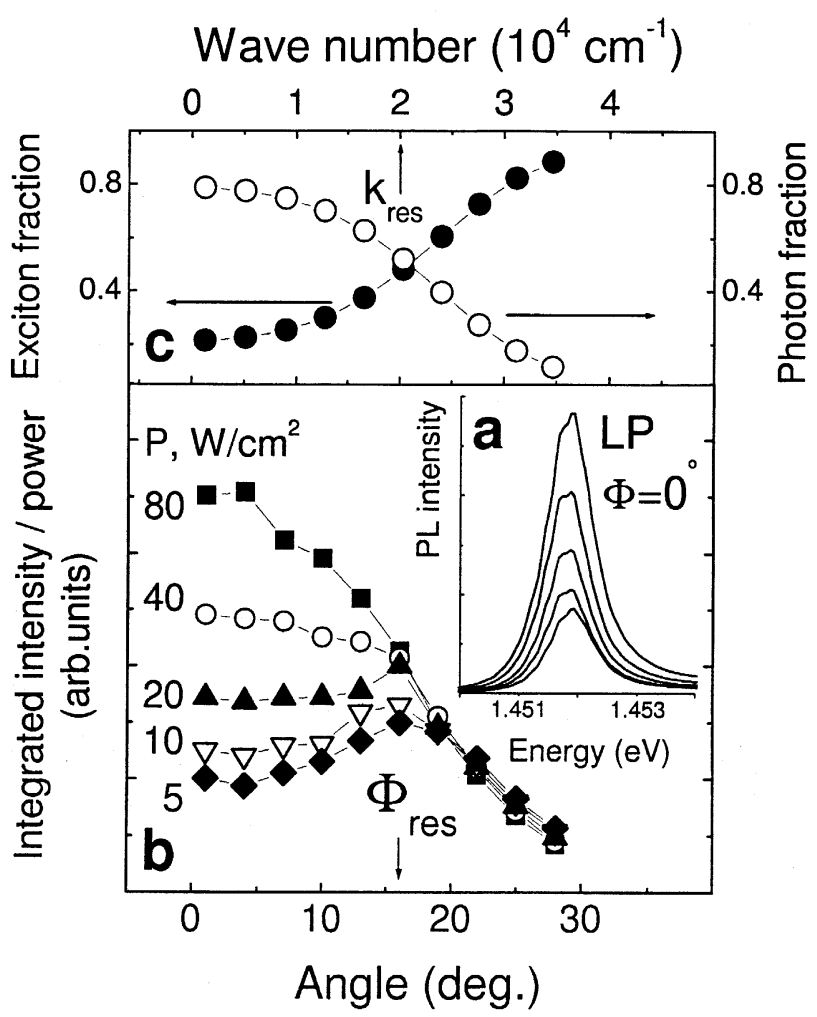

Fig. 4. (a) PL spectra normalised to the laser intensity for $P$ varying from 5 to $80 \mathrm{~W} / \mathrm{cm}^{2}$. (b) Integrated LP emission intensity divided by the laser power vs. angle for detuning $\Delta=-4.3 \mathrm{meV}$. (c) Photon and exciton fractions of the polariton states for $\Delta=-4.3 \mathrm{meV}$. 


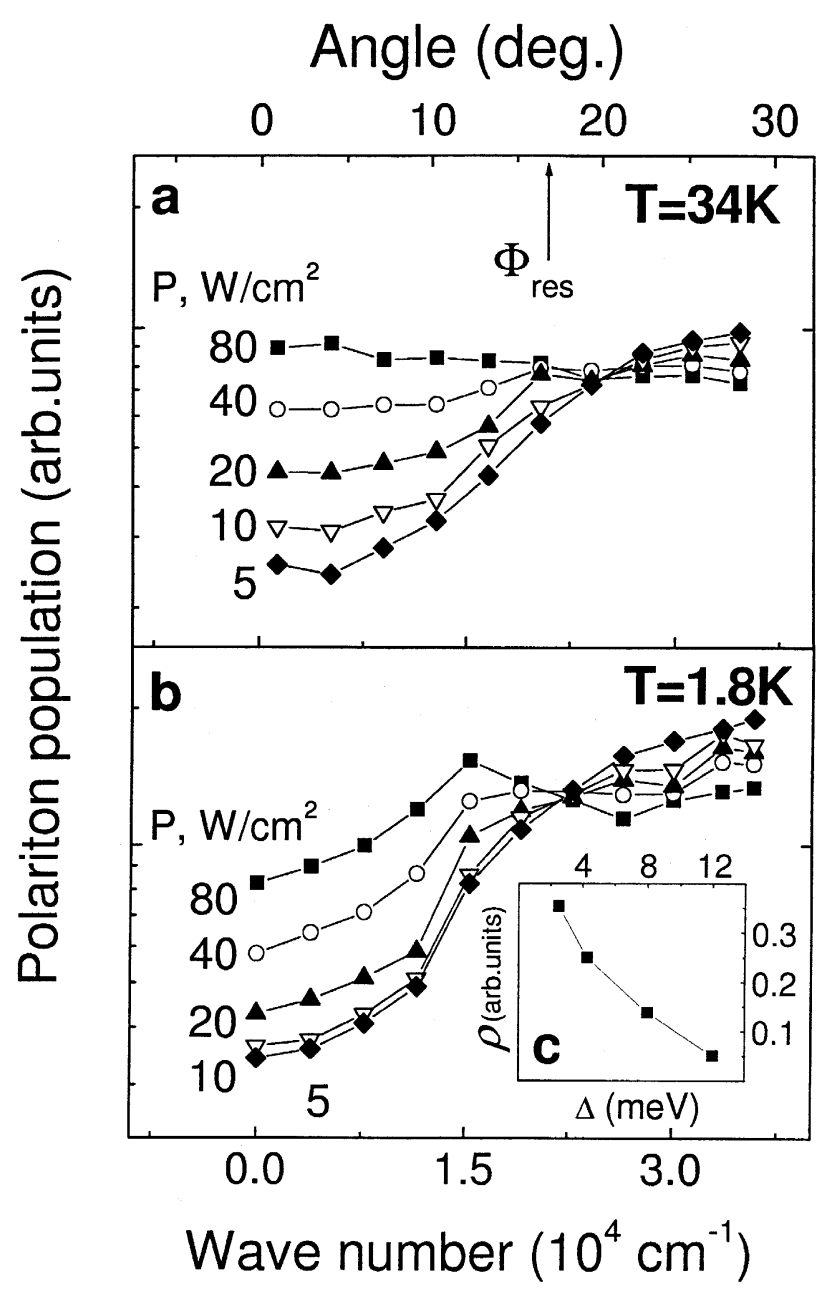

Fig. 5. Polariton population distribution for different excitation densities (a) $T=34 \mathrm{~K}$, (b) $T=1.8 \mathrm{~K}$. (c) Ratio of polariton populations $\rho=$ $N_{\mathrm{LP}}(0) / N_{\mathrm{LP}}\left(k_{\text {res }}\right)$ vs. $\Delta$ for low excitation and $T=1.8 \mathrm{~K}$.

tween acoustic phonon assisted polariton relaxation (1 ns timescale) from the exciton reservoir created by the nonresonant excitation, and the increasing escape rate from the cavity as the polariton states become increasingly photon like with decreasing $k$. Furthermore, and again importantly, for the higher power of $80 \mathrm{~W} / \mathrm{cm}^{2}$ the PL intensity by contrast peaks at $0^{\circ}$ and then decreases smoothly to higher angle, showing that the bottleneck is suppressed at higher powers [13].

These results are summarised in Fig. 4b where the PL intensities are plotted as a function of angle (and in-plane $k$ ). At low power, the PL intensity peaks at $16^{\circ}$ and decreases to lower and higher angle. With increasing power the bottleneck is steadily suppressed as a result of the increasing probability of polariton-exciton and polaritonpolariton scattering, until for powers greater than 40 $\mathrm{W} / \mathrm{cm}^{2}$ maximum intensity is observed from $\mathrm{k}=0$ states. The results in Fig. 4 can be converted into polariton population $N_{\mathrm{LP}}$ vs. $k$, since $N_{\mathrm{LP}} \approx I \tau_{\mathrm{LP}}$, where $\tau_{\mathrm{LP}}$ is the time for radiative loss from the cavity given by the photon lifetime in the cavity $(\sim 1 \mathrm{ps})$ divided by the photon fraction of the states involved (Fig. 4c). The resulting polariton populations vs. $k$ are shown in Fig. 5 where the bottleneck at low power and its suppression at high power are clearly seen.

Even more pronounced bottlenecks are observed for increasing negative detuning as shown by the results in Fig. 6 where $k=0$ PL intensities more than one order of magnitude smaller than at high angle are seen for a detuning of $-11.6 \mathrm{meV}$. This is a consequence of the very high photon fractions of the low $k$ states and hence much reduced relaxation probabilities to low $k$ before escape occurs from the cavity (the magnitudes of the bottleneck defined as the ratio of the $k=0$ population to that at high $k$ is shown as a function of increasing negative detuning are shown in Fig. 5c). In this case, the peak of the bottleneck shifts to lower $k$, but up to the powers employed in Figs. 3-5, the bottleneck is always observed. It is also notable that the bottleneck is not observed for zero and positive detunings, in contrast to theory predictions

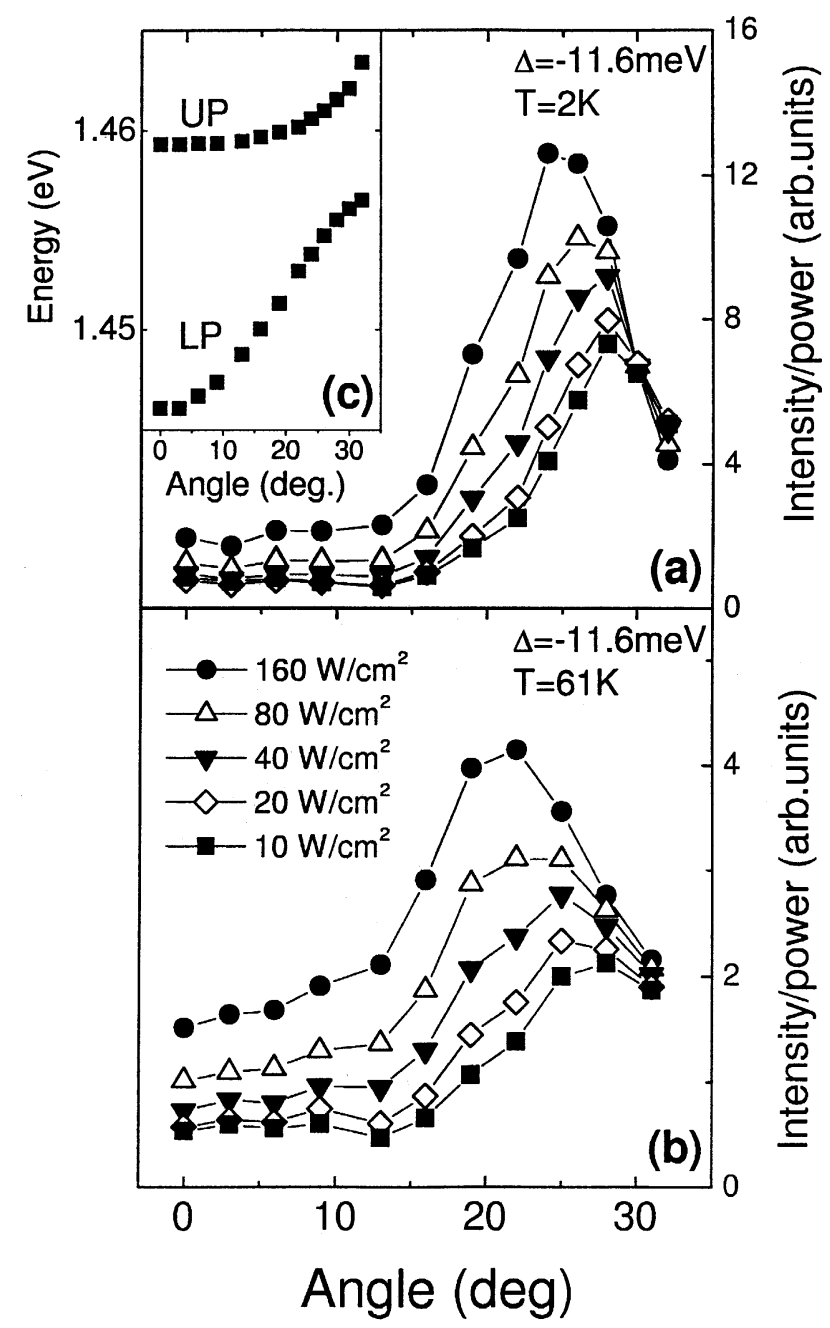

Fig. 6. (a) Integrated LP emission intensity divided by laser power vs. angle for detuning $\Delta=-11.6 \mathrm{meV}$ for powers from 10 to $160 \mathrm{~W} / \mathrm{cm}^{2}$ at $T=2 \mathrm{~K}$. (b) as in panel (a) but at $61 \mathrm{~K}$, (c) measured polariton dispersion. 


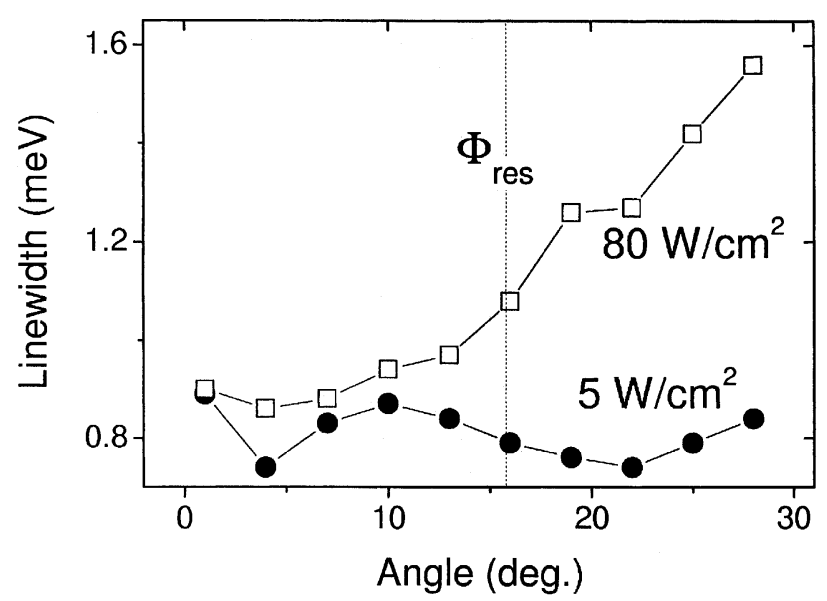

Fig. 7. Angular dependence of lower polariton branch PL linewidth for high (solid symbols) and low (open symbols) excitation power for $\Delta=-4.3 \mathrm{meV}$.

$[9,10]$. This is probably due to the omission of disorder scattering in the theory treatments where polariton scattering only by phonons and by excitons or polaritons is included.

Further information on the mechanism responsible for the suppression of the bottleneck with increasing power is obtained from study of the lower polariton branch linewidths as a function of $k$ at low and high power, as shown in Fig. 7. At low power, the linewidths are seen to be approximately independent of angle from $0^{\circ}$ to $30^{\circ}$, whereas at $80 \mathrm{~W} / \mathrm{cm}^{2}$ an increase in linewidth by a factor of two is observed over this range of angles due to the increased interparticle interaction with increasing density. This is consistent with the accompanying suppression of the bottleneck at similar powers due to polariton-polariton scattering which increasingly populates the low k states. Further pieces of evidence, not described in detail here, for the role of polariton-polariton scattering in suppressing the bottleneck are obtained from the greater suppression at a given power with increasing temperature, and from the maximum non-linear behaviour in the PL intensity found at an energy of $7 \mathrm{meV}$ below the uncoupled exciton energy, over a wide variety of detunings and temperatures [11]. The energy of $7 \mathrm{meV}$ cannot be explained by phonon-mediated processes since the maximum energy that can be transferred by phonons is $\sim 2 \mathrm{meV}\left(k_{\max } \sim\right.$ $2 \pi / l$, where $l$ is the well width). Its most likely origin is a scattering process where one exciton scatters down in energy from the reservoir and a second exciton (or polariton) scatters to higher energy either to the exciton continuum or to the upper polariton branch to conserve energy and momentum [11].

Finally, in this section we make two further remarks. The emission at $k=0$ shows marked super-linear increase with increasing laser power (seen clearly in Fig. 4a,b). However, there is no evidence for stimulation behaviour in the results; they can be explained purely in terms of exciton-exciton scattering processes. This conclusion was further supported by the results of Ref. [13], where the $k=0 \mathrm{PL}$ intensity was shown to increase quadratically with laser power, providing strong evidence for a pair scattering process leading to the suppression of the bottleneck. Further increase of power beyond that shown in Fig. 3 , leads to loss of strong coupling and conventional (photon) lasing in the system at the energy of the uncoupled cavity mode as shown in Ref. [3] and in Fig. 8b. The results of Ref. [11], which are supported by those of Ref. [13], show that previous claims $[14,15]$ of stimulated polariton scattering (boser behaviour [16]), at least in III-V cavities, under conditions of non-resonant excitation were premature.

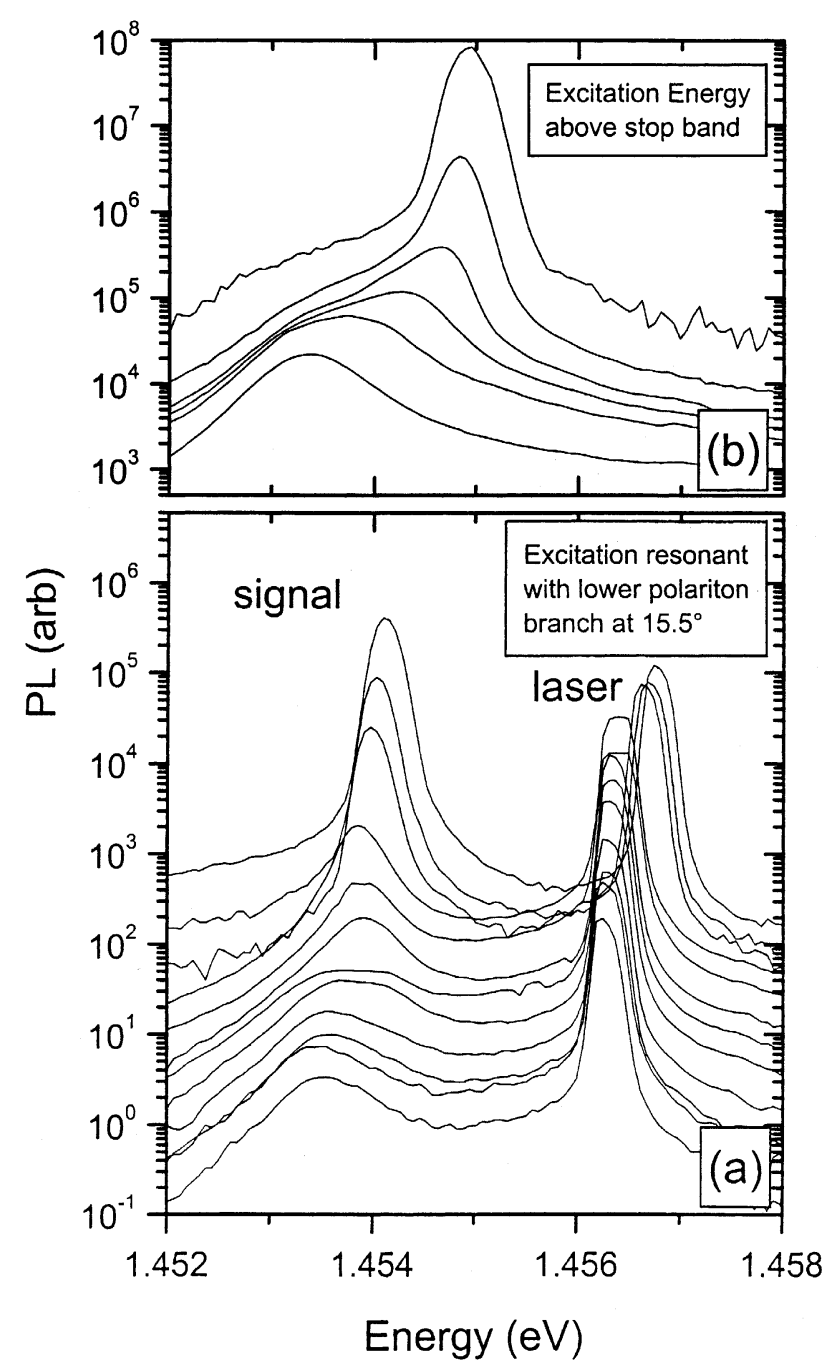

Fig. 8. (a) Photoluminescence spectra at $\theta=0^{\circ}$ for excitation resonant with the lower polariton branch at $15.5^{\circ}$, as a function of excitation intensity from 0.5 to $5 \times 10^{2} \mathrm{~W} / \mathrm{cm}^{2}$. The laser is retuned to higher energy to maintain resonance above the threshold of $\sim 150 \mathrm{~W} / \mathrm{cm}^{2}$ (fifth curve from top). (b) PL spectra as a function of excitation intensity for high-energy non-resonant excitation at $1.55 \mathrm{eV}$. By comparison with panel (a) where a small shift by $0.5 \mathrm{meV}$ to higher energy occurs above threshold, in panel (b) corresponding to conventional lasing in the weak coupling limit, a large shift by $1.5 \mathrm{meV}$ is observed. 


\section{Resonant excitation}

We now show that the situation is very different for conditions of resonant excitation of the lower polariton branch. Clear evidence for final state stimulation is obtained with the system remaining very clearly in the strong coupling regime. Strong line narrowing is found to accompany very strong, superlinear (near exponential) increases in $k=0$ intensity, characteristic of a process with gain, stimulated by transitions to a final state with macroscopic occupancy. Such final state stimulation can occur for any bosonic quasi-particle; the transition rate is proportional to $\left(1+N_{\text {final }}\right)$, where the 1 describes spontaneous processes, and $N_{\text {final }}$ describes stimulation of the transition by occupation of the final state. By contrast to fermionic particles, $N_{\text {final }}$ for bosons can be greater than one and gives rise, e.g. to stimulated emission in lasers where stimulation occurs when the occupancy of a photon mode of the cavity exceeds unity. ${ }^{1}$ Such stimulation behaviour is characteristic of any bosonic particle. Since excitons are bosons (although their constituent electrons and holes are fermions) and photons are bosons, polaritons are expected also to exhibit bosonic behaviour such as final state stimulation. The small polariton density of states arising from the small polariton mass, of order $10^{4}$ times smaller than for excitons [1], suggests that achievement of state occupancies greater than unity is likely to be very much easier than for excitons. The present experiments provide strong evidence for such behaviour.

PL spectra detected at $k=0$ as a function of laser power for the laser tuned to be in resonance with the point of inflection of the lower polariton branch are shown in Fig. 8a. The resonant excitation conditions are shown schematically on the $E-k$ diagram of Fig. $9 \mathrm{~b}$, where it is seen that excitation at $\sim 16^{\circ}$ permits pair (parametric) scattering to occur to higher and lower energy in a process conserving energy and momentum [17]. Above a threshold of $\sim 200 \mathrm{~W} / \mathrm{cm}^{2}$ the spectra show a very marked, nearexponential increase of intensity (see Fig. 8a), accompanied by line narrowing to a width of $0.2 \mathrm{meV}$, limited by the spectrometer resolution (subsequent experiments have shown a linewidth of $0.075 \mathrm{meV}$ in Ref. [7], and as small as $0.002 \mathrm{meV}$ in Ref. [5]). Accompanying the highly non-linear increase of intensity (Fig. 9a) and the line narrowing, the PL spectra shift by $0.5 \mathrm{meV}$ to higher energy and then remain fixed in energy to higher power, so long as the laser energy is also held constant. This behaviour is in marked contrast to that observed for non-resonant excitation in Fig. 8b. In this case, above the non-linear threshold the lasing peak occurs at $1.5 \mathrm{meV}$ above the energy of the lower polariton branch at low power, at an energy very close to that of the uncoupled cavity mode of

\footnotetext{
${ }^{1}$ To achieve lasing requires in addition that the gain should exceed the losses in the cavity.
}

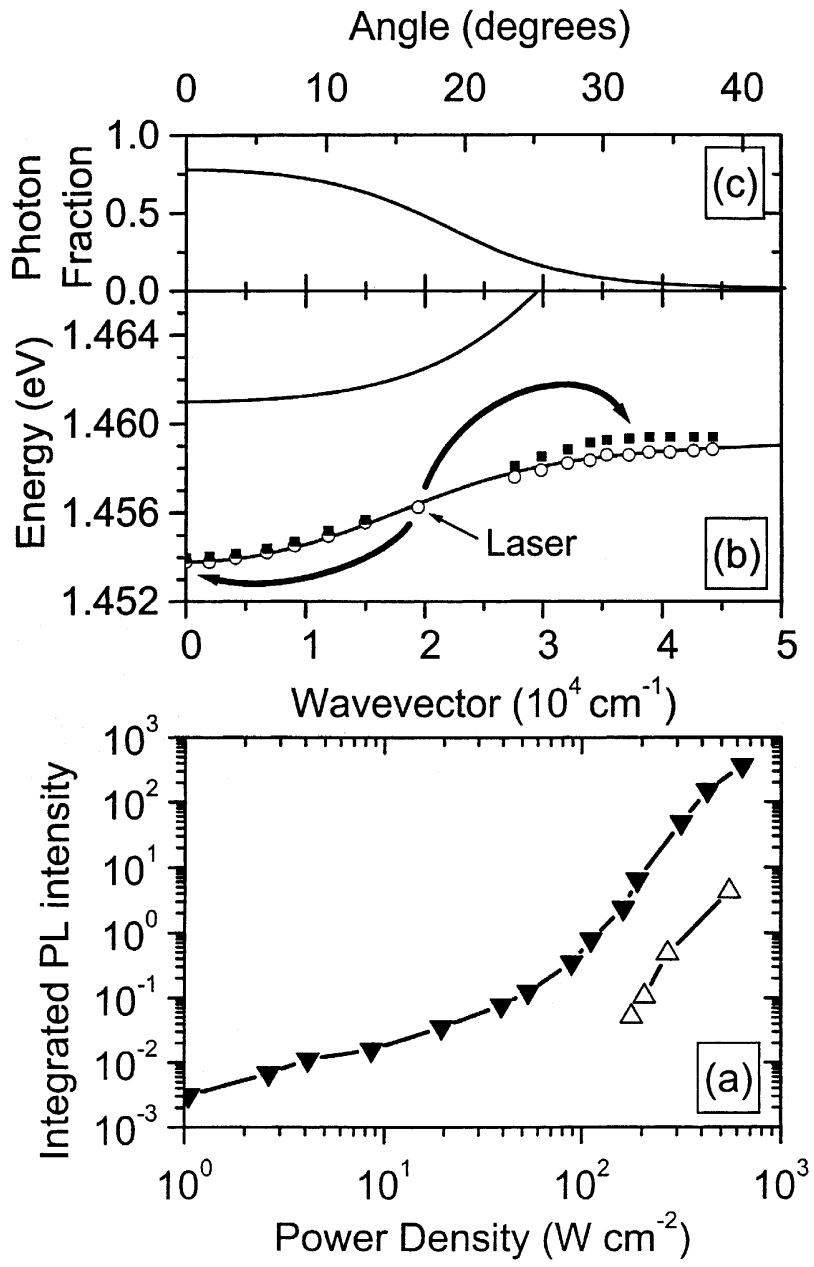

Fig. 9. (a) $k=0$ and $3.9 \times 10^{4} \mathrm{~cm}^{-1} \mathrm{PL}$ signal intensities vs. excitation intensity. Clear threshold behaviour is observed at a power density of 150 $\mathrm{W} / \mathrm{cm}^{2}$. (b) Measured lower branch dispersions below $\left(10 \mathrm{~W} / \mathrm{cm}^{2}-\right.$ open squares)) and above threshold ( $300 \mathrm{~W} / \mathrm{cm}^{2}$ - filled squares), together with fitted upper and lower branch dispersions. (c) Calculated photon fraction for lower branch as a function of $k$.

the system, thus showing that for non-resonant excitation the lasing occurs in the weak coupling limit.

The small shift in peak energy from below to above threshold for resonant excitation shows that the highly non-linear behaviour occurs in the strong coupling limit, and suggests strongly the involvement of polariton quasiparticles in the non-linear process. To investigate the behaviour further, angular-dependent measurements were performed to probe the polariton population distribution as a function of $k$ for laser powers below, close to and above threshold, as employed in the non-resonant case in Section 3 to reveal the existence of the relaxation bottleneck. The results are shown in Fig. 10.

At low power in Fig. 10a, the PL spectra are seen to peak in intensity around the laser angle and then to decrease in intensity to higher and lower angle (the measured integrated intensities vs. $k$ are shown in Fig. 11a). This is an example of the relaxation bottleneck but now observed 


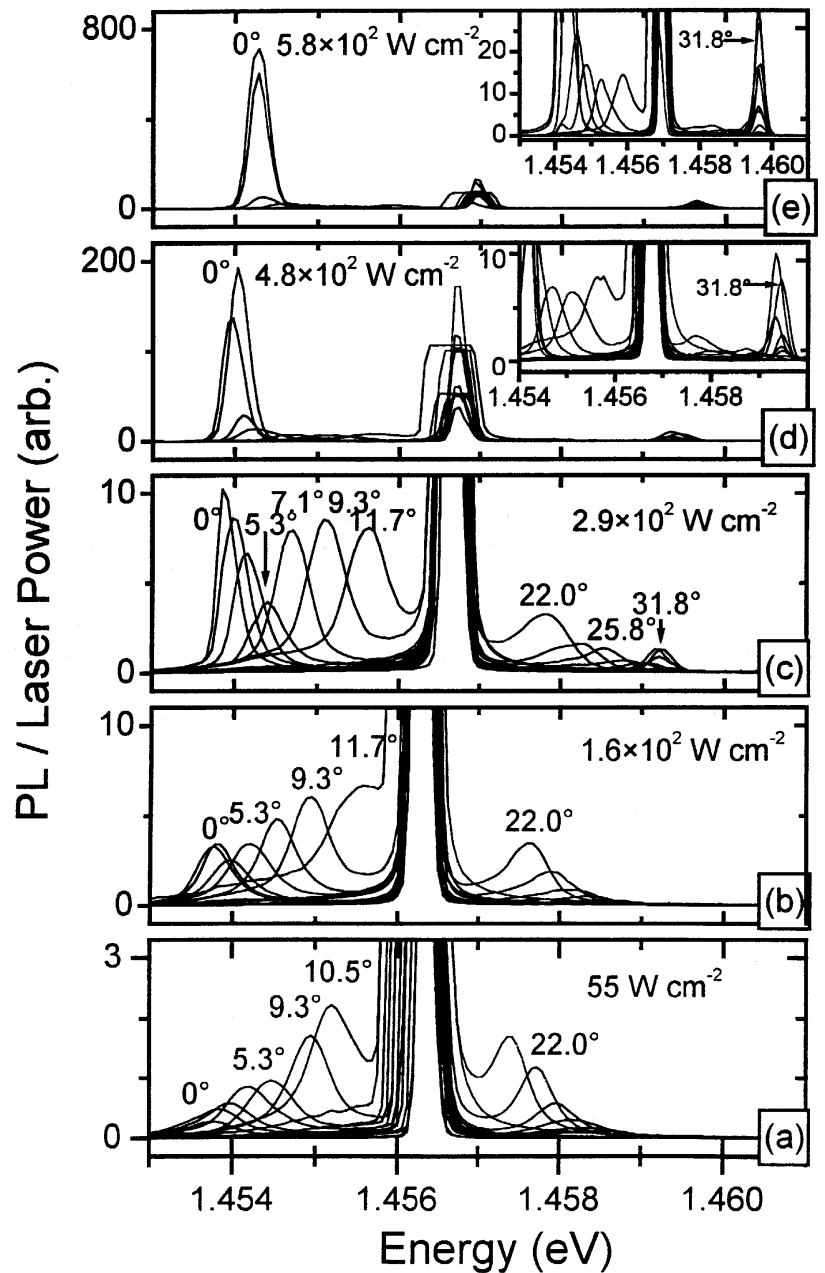

Fig. 10. PL spectra normalised to the laser power for excitation intensities labelled on the figure: (a) below, (b) close to threshold, and (c), (d), (e) above threshold as a function of detection angle from $0^{\circ}$ to $35^{\circ}$. A very strong change is observed between panel (a) at low power where the spectra are strongest for angles close to the excitation energy, and panels (d) and (e) where the spectra are dominated by two peaks at zero and at $32^{\circ}$. The very strong features at $\sim 1.456 \mathrm{eV}$ arise from the exciting laser at $15.5^{\circ}$.

for resonantly injected polaritons. At low powers, the polaritons undergo only weak polariton-polariton scattering, leading to small but detectable broadening of the injected population, before escape from the cavity occurs. With increasing power, the polariton distribution broadens further in $k$-space as the probability for parametric pair scattering increases, until close to threshold in Fig. 10c, the PL intensity is nearly uniform in $k$-space for $k<k_{\text {laser }}$, with a small peak developing at $k=0$. Further increase in laser power, now moving above the threshold of Figs. 8 and 9 , leads to a very strong increase of the $k=0$ intensity, accompanied by an additional strong peak at high $k$, the high $k$ component of the pair scattering process indicated schematically in Fig. 9, required to conserve energy and momentum in the process $2 k_{\text {laser }}=k_{\text {signal }}+k_{\text {idler }}$, $2 E_{\text {laser }}=E_{\text {signal }}+E_{\text {idler }}$, where the subscripts signal and idler refer to the $k=0$ and high $k$ beams, respectively. Furthermore, measurements of the polariton dispersion above threshold show that the polariton dispersion curve is only slightly modified from the case at low power (Fig. $7 b)$ and that the system remains clearly in the strong coupling limit. The angular-dependent measurements thus show that the strong non-linear behaviour observed for resonant excitation has a very different origin to that for non-resonant excitation, and that it arises from a polariton-polariton scattering process in the strong coupling regime to states at $k=0$ and high $k$.

The final step in elucidating the physics underlying the results of Figs. $8-10$ is to convert the PL intensities vs. $k$ of Fig. 11a to polariton population vs. $k$. Firstly, the relative PL intensities are converted to relative polariton occupancies by correcting the observed PL intensities by the photon fraction (and hence lifetime) of the states involved. Carrying out this correction leads to polariton occupancies in the signal and idler states within a factor of two of one another, as shown in Fig. 11b, consistent with the pair nature of the process which gives rise to the two

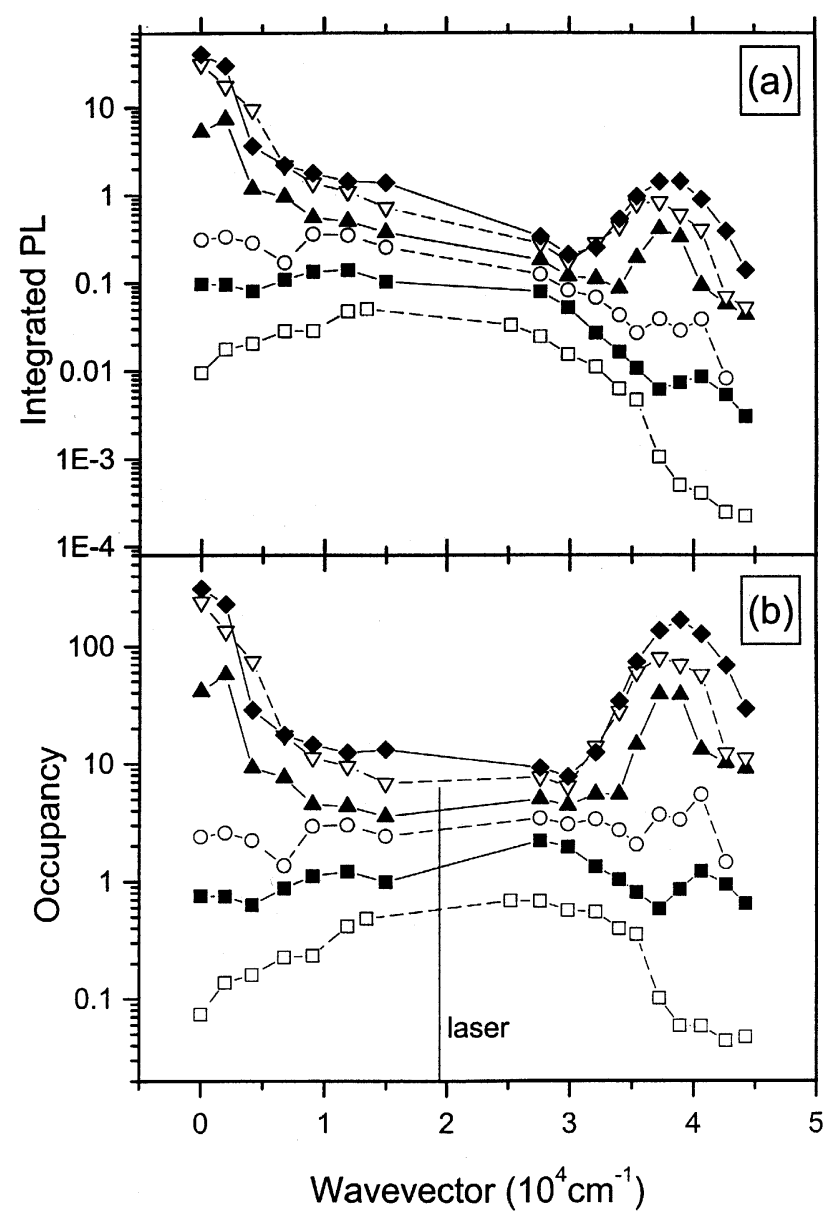

Fig. 11. (a) Integrated intensities of PL peaks as a function of in plane $k$ (angle); (b) same as panel (a) but corrected for the photon fraction of the lower branch as a function of $k$ (from Fig. 9c). 
beams. Finally, measurement of the absolute power $(P)$ emitted in the $k=0$ beam enables the occupancies $(N)$ to be placed on an absolute scale since $N=\left(I_{\mathrm{PL}} \tau_{\mathrm{pol}} 4 \pi\right) /$ $(\Delta k)^{2}$, where $I_{\mathrm{PL}}$ is the measured PL power in watts, $\tau_{\mathrm{pol}}$ is the polariton lifetime given by the photon lifetime in the cavity divided by the photon fraction of the $k=0$ state and $\Delta k$ is the width in $k$-space of the $k=0$ beam. Carrying out this procedure shows that the occupancy of the $k=0$ state at threshold is equal to unity within a total error of a factor of \pm 3 [3]. This is exactly the condition discussed at the start of this section necessary for the occurrence of final state stimulation of the pair scattering process. Taken together with the proof from the spectra and the dispersion above threshold that the signal beam arises from a system in the strong coupling limit, this result provides strong evidence for the occurrence of final state stimulation arising from the bosonic character of the polariton quasi-particles [18].

The scattering process to two specific points in $k$-space has many of the characteristics of a polariton condensation, to a final state with large occupancy, with the state occupancy reaching values greater than 100 at the highest powers employed. Furthermore, the strong line narrowing shows that the final state has macroscopic coherence. The spontaneous appearance of line narrowing above threshold shows that the polariton system has undergone a phase transition, consisting of the macroscopic occupation of a single mode. Since polaritons are bosons, there is some justification in describing this phase as a Bose-Einstein condensate (BEC). Support for this description is provided by clear observation of stimulated scattering of other polaritons into the macroscopically occupied mode, a characteristic property of a BEC. However, the system is far from thermal equilibrium, so although the initial build up of the $k=0$ population comes from scattering down from the pump population, the phase transition is not driven by cooling of the system. In this sense, it differs from the ideal picture of a BEC, which has a well defined temperature and a chemical potential approaching zero.

We first reported stimulated scattering behaviour in two-beam pump-probe experiments [4]. In this case a strong pump beam was incident on the sample at the point of inflection of the lower polariton branch. Very weak stimulation to $k=0$ was found with the pump beam alone present on the sample [6], probably because only a very small $k=0$ population builds up before all the photocreated polaritons escape from the sample. Injection of a second weak probe at $k=0$ was found to lead to very strong stimulated scattering from $k_{\text {laser }}$ to $k=0$ with very large gains being found. In the $\mathrm{CW}$ excitation experiments described here, by contrast the process is self-stimulated, with sufficient $k=0$ population building up to lead to strong stimulation without the need for injection of a second probe pulse.

It is instructive to compare these results with experiments performed over 20 years ago on bulk materials
$(\mathrm{CuCl})^{20}$, and termed by some of these authors hyper-Raman scattering. The main basic difference in the two cases is that in the $2 \mathrm{D}$ microcavity system the lower polariton branch has a finite energy at $k=0$ due to the photon confinement in the vertical direction (the waveguide cutoff), whereas in the bulk 3D case the lowest energy polariton has the slope of the photon light-line at small $k$, and its energy tends to zero as $k$ tends to zero. As a result in the microcavity case finite occupancy of the $k=0$ states occurs (the bottom of the polariton trap, see Fig. 2 inset) leading to stimulation of the scattering process to $\mathrm{k}=0$. Indeed in the microcavity case the incident laser, the signal and the idler are all resonant with real states of the system, accounting in part for the very small thresholds observed of order $100 \mathrm{~W} / \mathrm{cm}^{2}$ (as opposed to the $\mathrm{MW} / \mathrm{cm}^{2} \mathrm{em}-$ ployed in the bulk case) [19]. In an equivalent description to that employed here, the microcavity under resonant excitation can be regarded as a very low threshold optical parametric oscillator (OPO) [5] with triply resonant pump, signal, and idler beams, accounting for the very low thresholds compared to typical OPOs [20].

\section{Comparison of resonant and non-resonant excitation and conclusions}

The discussions in Sections 3 and 4 show that carriercarrier (pair) scattering processes (exciton-exciton, exciton-polariton and polariton-polariton) play a determining role in the polariton distributions which result from both non-resonant and resonant excitation of semiconductor microcavities.

In the non-resonant case at $\sim 4 \mathrm{meV}$ negative detuning, suppression of the bottleneck by polariton-polariton scattering is observed for power densities of $\sim 40 \mathrm{~W} / \mathrm{cm}^{2}$, with further increase of power to $\sim 150 \mathrm{~W} / \mathrm{cm}^{2}$ leading to lasing in the weak coupling limit. The strong coupling is lost due to exciton screening at carrier densities estimated to be $\sim 5 \times 10^{11} \mathrm{~cm}^{-2}$ (for a power density $100 \mathrm{~W} / \mathrm{cm}^{2}$ and assuming an exciton lifetime of $1 \mathrm{~ns}$ ), corresponding to an exciton density per well of $9 \times 10^{10} \mathrm{~cm}^{-2}$ [21], close to the Mott limit for excitons in quantum wells. Further clear evidence for the importance of exciton-exciton interactions was obtained from the marked increase of polariton linewidth for angles greater than $\sim 16^{\circ}$ in Fig. 6. However, polariton final state stimulation plays no role in the observed behaviour, with strong coupling being lost before $k=0$ occupancies become sufficiently large (greater than one) to initiate stimulation. The main difficulty with nonresonant excitation is that most of the photocreated carriers relax into the exciton reservoir with inefficient population of the small $k$ polariton states. Attempts to increase the low $k$ polariton population up to state occupancies close to unity leads to population of the exciton reservoir at densities greater than the exciton screening limit, with loss of strong coupling before stimulation occurs. 
The case of resonant excitation is very different. In this case, polaritons are injected into the polariton trap without direct population of the exciton reservoir. Polariton-polariton scattering leads to population of states at lower and higher k. Stimulation is observed at incident power densities of $\sim 200 \mathrm{~W} / \mathrm{cm}^{2}$, corresponding to a parametric process to pair states at $k=0$ and high $k$. Compared to the high energy non-resonant case where all incident photons are absorbed in the cavity, in the resonant case only $\sim 10 \%$ of the incident power is absorbed. Furthermore, and most importantly the lifetime of the injected particles is now given by the polariton lifetime (close to the photon lifetime of $\sim 1 \mathrm{ps}$ ), and the threshold is now achieved at a polariton density of only $\sim 10^{8} \mathrm{~cm}^{-2}$, well below the density for any significant screening. Hence, the strong coupling limit is retained at $k=0$ state occupancies in excess of unity, as shown in Section 4, permitting the occurrence of polariton final state stimulation.

Such a light emitter may reasonably be termed a polariton laser, although the stimulated process is the polariton scattering from the injection point to two specific points in energy and wavevector space. This gives rise to a $k=0$ polariton state with macroscopic occupancy, from which photon emission occurs as the polaritons are converted to external photons by leakage of their photon components through the Bragg mirrors. This situation is in marked contrast to a conventional laser where the photon emission process itself is stimulated, leading to macroscopic occupation of particular photon modes of the cavity.

In order to achieve polariton lasing under conditions of non-resonant excitation, it is necessary that $k=0$ occupancies greater than one be achieved before the strong coupling is lost due to exciton screening from the high density of excitons inevitably created in the exciton reservoir. However, if the strong coupling were retained the stimulation mechanism would then lead to very efficient scattering from the reservoir down to $k=0$, very efficient population of the emitting states, and at least in principle the basis of a new light source where injected carriers populate the 'lasing' state on an ultrafast timescale. The results presented in Section 3 show that such a situation cannot be achieved in GaAs-based III-V microcavities, due largely to the relatively small exciton binding energies $(\sim 8 \mathrm{meV})$ and correspondingly low densities for exciton screening.

II-VI [22], GaN and organic [23,24] microcavities all offer potentially more favourable systems for the achievement of polariton stimulation under non-resonant conditions, due to their significantly greater exciton binding energies (factors of 3 to 10 higher in the sequence II-VI, $\mathrm{GaN}$, organic). However, one possible drawback is that the greater exciton binding energies are accompanied by increased exciton oscillator strengths and hence normal mode splittings between upper and lower polariton branches also in the range of $\sim 3$ to 10 higher. As a result significantly greater energy loss will be required in order to achieve significant population of the $k=0$ states from the reser- voir, in part offsetting the gains due to resistance to screening. Precise experiments are required in order to answer the question as to which of the two processes, resistance to screening or greater energy loss proves to be more important. Nevertheless, it should be noted that some of the early experiments on II-VI microcavities did provide possible evidence for polariton lasing at intermediate excitation densities,[22] before collapse of the system to the weak coupling regime at higher powers, thus giving some optimism that such a polariton laser can be achieved in systems with large exciton binding energy. Finally, it should be noted that the resonantly excited microcavities may have considerable potential as highly efficient wavelength shifting devices, with energy shifts between laser and signal up to $5 \%$ of the pump energy possible in systems with large exciton oscillator strength such as organic semiconductor microcavities.

\section{Acknowledgements}

Support from EPSRC and EC Human Potential project Clermont (HPRN-CT-1999-00132) is gratefully acknowledged.

\section{References}

[1] For reviews, see

a M.S. Skolnick, T.A. Fisher, D.M. Whittaker, Semicond. Sci. Technol. 13 (1998) 645;

b G. Khitrova, H.M. Gibbs, F. Jahnke, M. Kira, S.W. Koch, Rev. Mod. Phys. 71 (1999) 1591

[2] C. Weisbuch, M. Nishioka, A. Ishikawa, Y. Arakawa, Phys. Rev. Lett. 69 (1992) 3314.

[3] R.M. Stevenson, V.N. Astratov, M.S. Skolnick, M. Emam-Ismail, A.I. Tartakovskii, P.G. Savvidis, J.J. Baumberg, J.S. Roberts, Phys. Rev. Lett. 85 (2000) 3680.

[4] P.G. Savvidis, J.J. Baumberg, R.M. Stevenson, M.S. Skolnick, J.S. Roberts, Phys. Rev. Lett. 84 (2000) 1547.

[5] J.J. Baumberg, P.G. Savvidis, R.M. Stevenson, A.I. Tartakovskii, D.M. Whittaker, J.S. Roberts, Phys. Rev. B 62 (2000) R16247.

[6] P.G. Savvidis, J.J. Baumberg, R.M. Stevenson, M.S. Skolnick, D.M. Whittaker, J.S. Roberts, Phys. Rev. B 62 (2000) R13278.

[7] A.I. Tartakovskii, D.N. Krizhanovskii, V.D. Kulakovskii, Phys. Rev. B 62 (2001) R13298.

[8] J. Toyozawa, Suppl. Prog. Theor. Phys. 12 (1959) 11.

[9] F. Tassone, C. Piermarocchi, V. Savona, A. Quattropani, P. Schwendimann, Phys. Rev. B 56 (1997) 7554.

[10] F. Tassone, Y. Yamamoto, Phys. Rev. B 59 (1999) 10830.

[11] A.I. Tartakovskii, M. Emam-Ismail, R.M. Stevenson, M.S. Skolnick, V.N. Astratov, D.M. Whittaker, J.J. Baumberg, J.S. Roberts, Phys. Rev. B 62 (2000) R2283.

[12] M. Müller, J. Bleuse, R. André, Phys. Rev. B 62 (2000) 16886.

[13] This result was also confirmed in P. Senellart, J. Bloch, B. Sermage, J.Y. Marzin, Phys. Rev. B 62 (2000) R16263.

[14] P. Senellart, J. Bloch, Phys. Rev. Lett. 82 (1999) 1233.

[15] S. Pau, H. Cao, J. Jacobson, G. Björk, Y. Yamamoto, Phys. Rev. A 54 (1996) R1789 (retracted in H. Cao et al., Phys. Rev. A55 (1997) 4632). 
[16] A. Imamoglu, R.J. Ram, Phys. Lett. A 214 (1996) 193.

[17] This process is described theoretically in

a)C. Ciuti, P. Schwendimann, A. Quattropani, Phys. Rev. B 63 (2001) R041303;

b)D.M. Whittaker, Phys. Rev. B 63 (2001) 193305.

[18] a) Further evidence for bosonic stimulation effects has been presented in Ref. [13] in two-beam experiments and in three-beam experiments in R. Huang, F. Tassone, Y. Yamomoto, Phys. Rev. B 61 (2000) R7854;

b) Highly non-linear behaviour in single-beam resonant excitation experiments has also been observed in R. Houdré, C. Weisbuch, R.P. Stanley, U. Oesterle, M. Ilegems, Phys. Rev. Lett. 85 (2000) 2793.

[19] See, e.g.

a) N. Peyghambarian, L.L. Chase, A. Mysyrowicz, Phys. Rev. B 27 (1983) 2325; b) J.B. Grun, B. Hönerlage, R. Levy, in: E.I. Rashba, M.D. Sturge (Eds.), Excitons, North Holland, 1982, p. 459.

[20] The terminology of signal and idler beams comes from the literature on OPOs, see e.g. A. Yariv, Quantum Electronics. Wiley, New York, 1988, p. 407.

[21] This density is similar to that obtained originally for the loss of strong coupling in microcavities by R. Houdré, J.L. Gibernon, P. Pellandini, R.P. Stanley, U. Oesterle, C. Weisbuch, J. O'Gorman, B. Roycroft, M. Ilegems, Phys. Rev. B 52 (1995) 7810.

[22] L.S. Dang, D. Heger, R. André, F. Boeuf, R. Romestain, Phys. Rev. Lett. 81 (1998) 3920.

[23] D.G. Lidzey, D.D.C. Bradley, T. Virgili, A. Armitage, M.S. Skolnick, S. Walker, Phys. Rfev. Lett. 82 (1999) 3316.

[24] A.I. Tartakovskii, M. Eman-Ismail, D.G. Lidzey, M.S. Skolnick, D.D.C. Bradley, S. Walker, V.M. Agranovich, Phys. Rev. B 63 (2001) R121-R302. 